\title{
Écrire l'histoire de la philosophie des Lumières
}

Note critique sur Bertrand Binoche «Écrasez l'infâme ! " Philosopher à l'âge des Lumières; Jonathan Israël Une révolution des esprits : les lumières radicales et les origines intellectuelles de la démocratie moderne; et Franck Salaün L'Autorité du discours : recherches sur le statut des textes et la circulation des idées dans l'Europe des Lumières Writing the history of Enlightenment philosophy. Critical analysis on Bertrand Binoche « Écrasez l'infâme! » Philosopher à l'âge des Lumières; Jonathan Israël Une révolution des esprits : les lumières radicales et les origines intellectuelles de la démocratie moderne; and Franck Salaün L'Autorité du discours : recherches sur le statut des textes et la circulation des idées dans l'Europe des Lumières

\section{Arnault Skornicki}

\section{OpenEdition}

\section{Journals}

Édition électronique

URL : https://journals.openedition.org/bssg/684

DOI : $10.4000 /$ bssg.684

ISSN : 2490-9424

Éditeur

Presses universitaires de Vincennes

Référence électronique

Arnault Skornicki, «Écrire l'histoire de la philosophie des Lumières », Biens Symboliques / Symbolic Goods [En ligne], 8 | 2021, mis en ligne le 20 mai 2021, consulté le 23 juillet 2021. URL : http:// journals.openedition.org/bssg/684; DOI : https://doi.org/10.4000/bssg.684 


\section{Écrire l'histoire de la philosophie des Lumières}

Note critique sur Bertrand Binoche « Écrasez l'infâme ! " Philosopher à l'âge des Lumières; Jonathan Israël Une révolution des esprits : les lumières radicales et les origines intellectuelles de la démocratie moderne; et Franck Salaün L'Autorité du discours : recherches sur le statut des textes et la circulation des idées dans l'Europe des Lumières Writing the history of Enlightenment philosophy. Critical analysis on Bertrand Binoche «Écrasez l'infâme! » Philosopher à l'âge des Lumières; Jonathan Israël Une révolution des esprits : les lumières radicales et les origines intellectuelles de la démocratie moderne; and Franck Salaün L'Autorité du discours : recherches sur le statut des textes et la circulation des idées dans l'Europe des Lumières

\section{Arnault Skornicki}

\section{RÉFÉRENCE}

Bertrand Binoche, «Écrasez l'infâme!» Philosopher à l'âge des Lumières, Paris, La Fabrique Jonathan Israël, Une révolution des esprits : les lumières radicales et les origines intellectuelles de la démocratie moderne, Marseille, Agone Franck Salaün, L'Autorité du discours : recherches sur le statut des textes et la circulation des idées dans l'Europe des Lumières, Paris, Honoré Champion 
"Écrasez l'infâme ! ", Une révolution des esprits, L'Autorité du discours

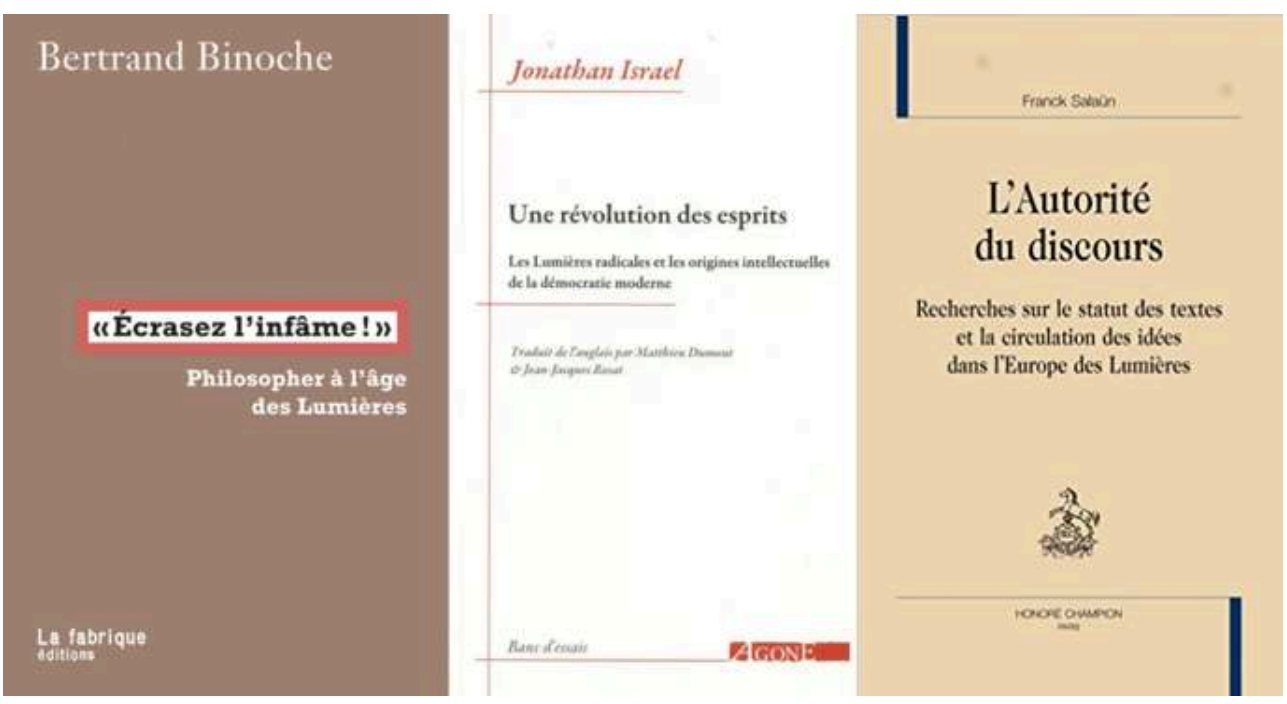

De gauche à droite : première de couverture de l'ouvrage de Bertrand Binoche (2018). «Écrasez

l'infâme! ». Paris, La Fabrique ; puis la première de couverture de l'ouvrage de Jonathan Israël (2017). Une révolution des esprits. Marseille, Agone; et enfin première la première de couverture de l'ouvrage de Franck Salaün (2010). L'Autorité du discours. Paris, Honoré Champion

(c) La Fabrique (c) Agone (c) Honoré Champion

1 Les discours publics sur les Lumières n'ont pas manqué ces dernières années, qu'ils prissent la forme de lieux communs, de polémiques politiques ou d'essais plus ou moins documentés. En revanche, les tentatives de définition rigoureuse des Lumières ou, du moins, de la philosophie des Lumières ne sont pas si fréquentes, même parmi les spécialistes. L'ouvrage d'Ernst Cassirer (2006 [1932]) reste une référence classique, mais ancienne, avec un net biais néo-kantien: s'il décline avec une grande force argumentative le triomphe de la raison analytique et de la philosophie expérimentale dans toute une série de domaines (science, histoire, politique, esthétique), il se fonde essentiellement sur l'étude d'une poignée de figures célèbres qui semblent préparer l'avènement de Kant. L'analyse de Peter Gay (1977), qui fait des Lumières le grand agent de la modernité démocratique et séculière en Occident, a été quelque peu démonétisée depuis que l'histoire sociale de la culture a largement revisité les Lumières au-delà de quelques cercles lettrés des capitales culturelles pour explorer leurs périphéries (comme les académies de provinces) et leurs canaux de diffusion, si l'on songe aux travaux pionniers de Daniel Roche, Robert Darnton ou Roger Chartier. Enfin, les propositions de Michel Foucault (1984) sur les Lumières comme ethos philosophique plutôt que contenu doctrinal, geste critique et ontologie de nous-même plutôt que rationalisme humaniste, sont aussi célèbres que discutées : toutefois elles restèrent à l'état de simples pistes et suggestions. Entretemps, une littérature scientifique pléthorique a pris un essor international jamais démenti, mais qui tendit à éparpiller les Lumières dans une foule d'études pointues et dispersées entre plusieurs disciplines (histoire, lettres, philosophie, esthétique, langues et civilisation...).

2 Toutefois, trois tentatives récentes pour mettre de l'ordre dans ce maquis méritent d'être relevées. Elles abordent le problème différemment en fonction des compétences et des appartenances disciplinaires respectives de leurs auteurs. Celle de l'historien Jonathan Israël, spécialiste mondialement célèbre (et controversé) des Lumières, peut se lire comme le vade-mecum d'une œuvre d'ores et déjà monumentale d'histoire 
intellectuelle. La deuxième, celle de l'historien de la philosophie Bertrand Binoche, repose sur une démarche diamétralement opposée, clairement nominaliste et antiessentialiste. La dernière, celle de l'historien de la littérature (mais aussi de la philosophie) Franck Salaün, apparaît moins systématique que les précédentes, mais plus proche de la sociologie des œuvres.

\section{Les idées radicales ont-elles fait la Révolution?}

3 Il n'est pas excessif de qualifier à la fois de substantialisme et d'antagonisme la démarche de Jonathan Israël. Les mérites de ce dernier sont d'importance. À l'origine spécialiste des Provinces-Unies, il a contribué au décentrement de l'étude des Lumières des nations dominantes sur les plans culturels et historiographiques (la France, le Royaume-Uni, dans une moindre mesure l'Italie), en faisant la part belle à l'Allemagne, la Hollande, ou l'Amérique du Nord. Son incontestable érudition est servie par une remarquable polyglossie qui lui donne accès à un impressionnant éventail de sources européennes et nord-américaines. Il a donné une inédite extension chronologique aux Lumières, peut-être excessive ( $\mathrm{du}$ milieu $\mathrm{du} \mathrm{xvII}^{\mathrm{e}}$ siècle aux grandes révolutions démocratiques de la fin du siècle suivant), mais qui permettait de questionner le rabattement des Lumières sur le seul XviII ${ }^{\mathrm{e}}$ siècle. Il a tenté de mettre au jour un courant souterrain, voire clandestin, des Lumières, celui du spinozisme (Israël 2005, $2008,2011)^{1}$. Même si cette découverte n'était pas entièrement inédite, même si l'auteur forçait déjà beaucoup la construction du corpus et s'exposa dès le départ à de fortes critiques $^{2}$, il incita à jeter le regard au-delà du mythe des Lumières bourgeoises pour interroger les "Lumières radicales ${ }^{3}$ " et à mettre en avant une série d'auteurs qui défendaient le matérialisme athée, la démocratie, l'égalité ou la justice sociale. Toutefois, l'évolution quelque peu dogmatique de ses travaux a montré toutes les limites de sa thèse séminale. Celle-ci est connue. Avec les Lumières, l'histoire des idées politiques - et même l'histoire tout court - est animée par un conflit fondamental entre deux courants. Le courant modéré associe déisme, spiritualisme et providentialisme d'une part, et de l'autre conservatisme sociopolitique ou, au mieux, réformisme superficiel. Le courant radical associe quant à lui matérialisme et athéisme d'un côté, positions démocratiques, anti-esclavagistes et égalitaires, voire révolutionnaires de l'autre. Reste que la thèse plus spécifique d'Une révolution des esprits concerne les origines idéologiques de la Révolution française. Cette dernière recevrait pour précondition nécessaire une "révolution des esprits » que les philosophes radicaux auraient opérée les décennies précédant 1789. Sans plus de détails ni de références historiographiques, l'auteur prétend s'appuyer sur les données de l'histoire du livre qui aurait prouvé la large diffusion des Lumières radicales (en particulier le baron d'Holbach ou l'abbé Raynal) à la fin de l'Ancien Régime. En défendant l'idée que les livres font bel et bien les révolutions, il s'inscrit explicitement contre la thèse de Roger Chartier selon laquelle c'est plutôt la Révolution qui a fait les Lumières et son canon de grands philosophes. Pourtant, Jonathan Israël ne donne d'autre preuve de cette " révolution des esprits» que ses effets supposés ex post. Autrement dit, il part du résultat final (la grande Révolution) pour remonter le fil de l'histoire. Or, on sait que seule une minorité de députés révolutionnaires étaient imbibés d'idées des Lumières, radicales ou non (Tackett 1997). Non pas que celles-ci n'aient joué aucun rôle dans le processus révolutionnaire, comme le montre l'exemple éloquent de la Déclaration des droits de l'homme et du citoyen: mais elles firent l'objet de réappropriations 
stratégiques dont il convient de déterminer précisément les circonstances et le poids dans le comportement des acteurs. C'est la dynamique de la crise politique qui a dicté des choix idéologiques que la plupart des membres de l'Assemblée constituante n'avaient nullement en tête au départ ${ }^{4}$.

Il convient, avance le préfacier du livre (Jean-Jacques Rosat), de «prendre les idées au sérieux ». Une telle affirmation ne devrait pas heurter outre mesure les spécialistes d'histoire des idées et représentations attentifs'ives à la formation, la transformation et la circulation des formes symboliques, celles-ci jouant un rôle clé dans les processus de légitimation et délégitimation de l'ordre social. La conclusion méthodologique que Jonathan Israël en tire est en revanche plus contestable. Elle se trouve bien éloignée du matérialisme qu'il encense pourtant, ou de son héros Spinoza qui déclinait un véritable programme d'analyse des œuvres dans le septième chapitre du Traité théologicopolitique. Les hommes (car - en dehors d'une mention de Mary Wollstonecraft - il s'agit pour l'essentiel d'hommes dans ce récit) sont les supports de concepts majestueux, mieux, de grandes luttes entre visions antagonistes du monde qui se révèlent dans l'histoire sous différentes déclinaisons, variantes et variations. L'inspirateur secret de l'auteur n'est-il pas, plutôt que Spinoza, Arthur Lovejoy, pour lequel l'histoire intellectuelle est faite d'«idées élémentaires" (unit-ideas) transhistoriques, qui se composent et se coagulent diversement à travers le temps sans jamais changer ellesmêmes? Une fois admise cette profonde conflictualité entre modération et radicalité qui animerait l'histoire des idées, tout le travail de l'historien consisterait alors à classer les auteurs sous l'une ou l'autre catégorie. Cette démarche entraine bien des risques. Le premier est de figer les œuvres dans un dualisme sans nuance, voire un manichéisme naï : pas même un continuum mais, dans les termes de l'auteur, « une dichotomie sans conciliation possible (...) une bipolarisation à laquelle personne ne pouvait échapper " (Israël $2017: 36$ ). Cette «bipolarisation" conduit tout droit à des lectures mutilées et simplificatrices, quand les contradictions et les ambiguïtés des Lumières sont légion, parfois chez le même auteur : ainsi Jonathan Israël accomplit le tour de force de parler beaucoup de Diderot sans guère mentionner ses contradictions continuelles; de donner une vision tout à fait simpliste de l'économie politique des Lumières, assimilée à une apologie du libre-échange alors qu'elle fut constamment parcourue de vives controverses ; d'affirmer tout de go que l'abbé Galiani (l'économiste et diplomate napolitain) est un avocat de l'égalité, etc. Last but not least, l'embarras manifeste de l'historien à propos de Rousseau prouve à lui seul les difficultés dans lesquelles il s'est lui-même placé, puisqu'il est bien en peine de classer le Citoyen de Genève dans le dispositif qui est le sien : ce dernier condamnait en effet sans ambiguïté le matérialisme tout en défendant les idées républicaines de liberté et d'égalité. Paradoxe ultime, sa critique du gouvernement représentatif, loin de lui être comptée comme un élément de radicalité, est plutôt rangée par Jonathan Israël comme un archaïsme suspect.

5 Le second risque, évidemment, est de rétro-projeter des classifications politiques sur des agents qui ne pouvaient pas en avoir la moindre idée. Prenons l'exemple de la bête noire de Jonathan Israël : Voltaire, figure typique des Lumières modérées, qu'il n'est pas loin de portraiturer en courtisan bien-pensant au point qu'on se demande parfois ce qui le sépare vraiment des adversaires du "parti philosophique ». D'une part il est étrange d'accuser Voltaire de s'être commis avec les puissants quand certains des héros du livre, tels Helvétius (un opulent fermier général) ou d'Holbach (un ancien avocat au 
parlement de Paris, issu d'une riche famille allemande), appartiennent eux-mêmes aux élites aristocratiques de leur temps et, de ce fait, sont protégés par leur fortune et leur statut. D'autre part, c'est ignorer que Voltaire comptait aussi des ennemis acharnés parmi les élites dominantes. Nombreux, au sein de l'Église et chez les parlementaires jansénistes (qui s'illustrèrent dans les affaires Calas et de La Barre), souhaitaient lui faire payer sa fameuse déclaration de guerre contre la superstition: "Écrasons l'infâme!", qui donne le titre à l'ouvrage de Bertrand Binoche. Antoine Lilti faisait remarquer à juste titre que cet usage public de la philosophie ne manquait pas d'une forme de courage et même, quoi qu'il en soit du conservatisme politique de Voltaire, d'une forme de "radicalité» (Lilti 2009: 197). Autrement dit, ce n'est pas tant l'anachronisme de la notion de radicalité qui pose problème que la manière dont Jonathan Israël l'a construite, entièrement autour du matérialisme athée et de la défense de la démocratie représentative, se rendant ainsi aveugle à d'autres formes d'intransigeance intellectuelle et d'engagement politique.

\section{Le régime intellectuel des Lumières : pratiques philosophiques, intertextualité et réseaux}

6 Tout autres sont les démarches de Bertrand Binoche et de Franck Salaün. En dépit de différences notables dans leurs modes de contextualisation (les débats philosophiques dans un cas, les réseaux et l'intertextualité dans l'autre), elles entretiennent de véritables affinités. Aucune ne part d'une définition présupposée des Lumières, ni ne cherche à classer artificiellement les œuvres. Chacune épouse autant que possible les sources, le mouvement des textes et des argumentations, considérés non comme l'expression de l'esprit du temps, mais comme l'agencement différentiel de prises de position. Ainsi, en historien de la philosophie, Bertrand Binoche se fonde-t-il sur un corpus certes resserré d'auteurs français, écossais et allemands, mais examinés en profondeur et parfois aux marges du canon philosophique: Nicolas Boulanger ou Charles de Brosses, par exemple, mais aussi Voltaire lui-même, central dans les études littéraires mais bien mal considéré dans le champ philosophique. Bertrand Binoche s'est permis de reprendre à son compte l'ambitieuse question à laquelle Kant avait donné une réponse célèbre en 1784 : qu'est-ce que les Lumières ? Pour se faire, il prend l'exact contrepied de Jonathan Israël: nulle ne saurait donner une définition substantielle des Lumières en raison de l'irréductible pluralité des thèses qui furent soutenues, tant sur les plans politique et moral qu'épistémologique ou esthétique. Celles et ceux qui ont tenté de dégager une essence commune en sélectionnant les traits réputés saillants des Lumières n'ont en général fait que reproduire un catéchisme scolaire issu de la Révolution et de la III ${ }^{e}$ République : philosophie expérimentale, droits naturels de l'homme, tolérance, croyance au progrès, anti-absolutisme, libéralisme, humanisme, amour de la raison... Or cette canonisation ne correspond guère à la belle diversité voire à la conflictualité des hommes et des femmes des Lumières.

7 En sautant à pieds joints dans leur contenu philosophique, il propose une autre approche, soustractive et non additive. Qu'est-ce qu'il reste des Lumières une fois retirées leur pluralité, leurs tensions et luttes internes? Non pas une doctrine ni une "philosophie ", mais un "philosopher », un style de pensée indocile et critique. S'il rejoint Kant et Foucault dans cette caractérisation générale des Lumières, Bertrand Binoche s'en démarque sur deux points. Contre le premier, la critique apparaît comme 
un exercice foncièrement collectif, et non l'effort du seul sujet pour penser par soimême. Hommes et femmes des Lumières partagent le geste de la critique: non pas exclusivement au sens kantien, mais au sens général d'une disposition collective à lutter contre les préjugés et la superstition ; à lutter, en somme, contre ce que Bertrand Binoche ramasse sous le concept générique (emprunté à Nietzsche) de "prêtre ». À la différence du second, il désigne clairement l'adversaire qui fédère les Lumières: la "superstition» et le "préjugé ». On pourrait croire qu'il s'agit là d'un banal anticléricalisme. Mais les Lumières visent au-delà du pouvoir institutionnel de l'Église. Leur plus petit dénominateur commun n'est pas la plus minuscule de leur facette : au contraire, cette disposition critique en constitue le fil conducteur et permet de revisiter - outre les penseurs susmentionnés - Denis Diderot, Jean-Jacques Rousseau, David Hume, Adam Ferguson, William Robertson, Emmanuel Kant, etc. Cette lutte contre la prétention des autorités religieuses à monopoliser le savoir et la morale légitimes fut au fondement de la «coalition » des philosophes (au sens d'une alliance défensive et offensive). Dès lors, « les Lumières se définissent par une discussion sur le sens exact qu'il s'agit de conférer à cette adversité » (Binoche 2018: 51). L'auteur explore avec acrobatie cette discussion souvent conflictuelle dans ses moindres recoins argumentatifs.

8 Cette définition négative recouvre toutefois une certaine positivité sur le plan philosophique. Cette positivité ne concerne pas le contenu des thèses, mais la manière d'exercer la philosophie comme activité critique de la superstition et de ses doubles intellectuels (le préjugé et la providence) et politique (l'esclavage, au sens générique). Par rapport au doute radical de Descartes, la spécificité des Lumières fut de collectiviser la critique et de la mettre au cœur de l'espace public, en une sorte d'opération générale de dé-dogmatisation dont l'Encyclopédie constitue la pierre de touche. Cette dédogmatisation n'exclut pas la spéculation, comme l'histoire hypothétique de Discours sur l'inégalité (1755) de Rousseau ou les Pensées sur l'interprétation de la nature (1753) de Diderot, ni les beaux "systèmes " (il y en eut à foison au XVIII ${ }^{e}$ siècle). Toutefois, selon Bertrand Binoche, les Lumières élaborèrent une conception du savoir qui exclut Dieu en tant que «formidable pétition de principe» (Binoche $2018: 64$ ). Cette revendication d'autonomie épistémique est une claire déclaration publique d'indépendance à l'égard de l'emprise de la théologie. L'analyse, puissamment étayée, est convaincante. Elle appelle cependant une réserve: ce combat contre "l'infâme » semble caractériser avant tout la France, où l'Église catholique continuait de revendiquer l'hégémonie culturelle. Il ne semble pas aussi central dans les Lumières britanniques, qui s'articulent parfois avec des formes contestataires de religiosité comme dans les cas de Joseph Priestley (à la fois pasteur et éminent chimiste, matérialiste et providentialiste, favorable aux révolutions française et américaine...), ou Benjamin Lay (le célèbre Quaker abolitionniste) (Rediker 2019).

9 Par sa forme même, la contribution de Franck Salaün refuse également toute substantialisation des Lumières. Pointilliste et érudite, elle se présente comme une série d'études de cas minutieuses et offre un tableau suggestif qui ne prétend jamais définir les Lumières - même négativement. Cependant, il rend sensible la force des réseaux, des sociabilités et des hostilités qui tissent leur "philosophie ». Le sous-titre indique à lui seul une proximité avec la sociologie historique des idées : Recherches sur le statut des textes et la circulation des idées dans l'Europe des Lumières. Ce travail offre en effet " une réflexion sur les discours en tant que pratiques », le " coefficient de vérité » qui détermine leur autorité et leur performativité. Les sources sont essentiellement 
imprimées, comme celles de Jonathan Israël et de Bertrand Binoche, mais leurs genres sont plus variés : philosophie, littérature, pamphlets, périodiques, correspondances. Les auteurs canoniques y côtoient des références moins familières (Le Pour et Contre, le journal de l'abbé Prévost qui eut toutefois son heure de gloire; Boureau-Deslandes et ses Réflexions sur les grands hommes qui sont morts en plaisantant, 1714), voire assez confidentielles (les abbés Dufour ou Gauchat). Marivaux, qui hante une grande partie de l'ouvrage, y est traité obliquement, comme penseur plus que comme dramaturge.

Écartant la notion d'«esprit » des Lumières, Franck Salaün adopte résolument une perspective relationnelle. Il part des tentatives d'autodéfinition de leur époque par les acteurs lettrés eux-mêmes ("siècle éclairé, siècle philosophe»), reconstitue les controverses entre philosophes et antiphilosophes, et plus largement les réseaux de discours et pratiques qui leur donnent consistance, en particulier dans la dernière partie («Système du discours et république des lettres »). Il reprend ainsi le dossier classique des « frères ennemis » Rousseau et Diderot dans une analyse fort éclairante de leur interdépendance intellectuelle, relative à leurs positions différentielles dans ce qui ne saurait être qualifié de champ intellectuel. Par exemple, le célèbre concept de perfectibilité, inventé par Rousseau, est mis à l'épreuve par Diderot au sein d'un espace de débats - dont le premier a largement contribué à tracer les contours - à propos des origines et des effets de la civilisation sur les mœurs. L'étude de leur intertextualité répond aux tumultueuses variations de leur rivalité que dévoile leur correspondance. De même, l'année qui suit la publication de La nouvelle Héloïse, Rousseau publie coup sur coup l'Émile et $\mathrm{Du}$ Contrat social. L'année 1762 est ce moment-charnière que Franck Salaün choisit d'étudier non à partir des œuvres mêmes, mais " en coupe ", sur la base de sa correspondance (dont l'édition est désormais complète et scientifiquement établie). Celle-ci révèle comment l'inscription dans son réseau structure ses stratégies éditoriales et de présentation de soi. Son ambition de s'affranchir des grands entre en tension avec son allégeance au prince de Conti. Ses discussions avec les éditeurs et ses négociations avec Malesherbes (le directeur de la Librairie, qui refusera la permission tacite au Contrat social) indiquent les contraintes sociopolitiques qui pèsent sur lui : l'Émile est condamné, son auteur est décrété de prise de corps par le Parlement de Paris puis par le Conseil de Genève. Si sa notoriété le protège, elle le menace aussi, et elle l'épuise, sous l'effet des innombrables sollicitations d'admirateurs et admiratrices dont il fait l'objet. Son nom d'auteur lui échappe au point que "Rousseau» devint un "phénomène culturel », voire « un ensemble de rapports et de croyances ». Franck Salaün appelle ainsi à recourir à l'outil informatique pour mener une étude réticulaire de la correspondance de Rousseau, afin de mieux situer la série successive de ses prises de position «en fonction de différents rapports de force » (Salaün 2010 : 311-312).

\section{Deux concepts à l'épreuve : providence et matérialisme}

11 Pour prendre la mesure plus précise des différences de méthodes entre nos trois auteurs, deux concepts doivent retenir l'attention parce qu'ils sont au cœur de l'interprétation globale de Jonathan Israël, et que le premier est traité par Bertrand Binoche, le second par Franck Salaün. L'histoire d'abord, ou plus exactement la providence. Fort de son expertise pointue (Binoche 2007, 2013, 2018), Bertrand Binoche 
montre que, si « philosophie de l'histoire » il y a dans les Lumières (l'expression est de Voltaire), celle-ci est rarement indexée à un progrès qui serait l'agent de l'histoire, et encore moins à une intervention divine. C'est bien plutôt le contraire : non seulement l'adversaire commun des "philosophes » reste précisément l'histoire universelle et providentielle de Bossuet, mais la plupart cherchent à conjurer autant que possible toute perspective téléologique dans le récit de l'histoire humaine, fût-elle sécularisée. S'il peut y avoir du progrès dans l'histoire, il ne saurait y avoir de progrès de l'histoire. À nouveau, le cas de Voltaire illustre bien le conflit d'interprétations. Jonathan Israël le range sans nuance du côté des défenseurs de la Providence, au prétexte que le philosophe de Ferney persistait à voir quelque finalité dans la perfection de la nature. Toutefois, il est probable qu'il s'agissait d'une tactique destinée à réduire la Providence aux lois de la nature établies par Newton, et à retrancher Dieu derrière les étoiles, loin de l'histoire des hommes (Binoche 2019 : 113). Bertrand Binoche n'a, ainsi, nulle peine à montrer que le monumental Essai sur les mœurs (1756) - véritable histoire globale avant la lettre - n'avait d'autre but que de montrer que la seule loi de l'histoire est de n'en avoir point; que le mal n'est pas toujours puni et que la justice en semble singulièrement absente; que, jusqu'à la Renaissance au moins, elle est dominée par le chaos des passions humaines. Entre les mains de Voltaire, l'histoire devient une remarquable machine de guerre contre le providentialisme. C'est donc un tout autre tableau qui ressort des analyses de Bertrand Binoche.

Le second concept est le matérialisme, dont Jonathan Israël fait la ligne de partage entre modération et radicalité. Il suppose d'une part une définition univoque dudit matérialisme (l'esprit est dérivé de la matière), d'autre part qu'entre les principes épistémologiques et les orientations politiques, la conséquence est toujours bonne. Sur ce dernier point, rien n'est moins certain, même si l'on peut admettre que les philosophes réputés matérialistes $\mathrm{du} \mathrm{XVIII}^{\mathrm{e}}$ siècle penchaient plus que d'autres pour des formes démocratiques de pouvoir. Cela n'a pas toujours été le cas: les «libertins » et autres «esprits forts " du siècle précédent penchaient plutôt, quant à eux, pour la raison d'État et la monarchie absolue. Et, au siècle des Lumières, quelques philosophes défendaient des formes d'utopie démocratique et de communauté des biens sans être tous matérialistes, loin s'en faut ${ }^{5}$. Contrairement à Jonathan Israël, qui se dispense de toute enquête sur ce que le matérialisme (ou le radicalisme, au demeurant) voulait dire pour les acteurs de l'époque, Franck Salaün se montre très attentif aux usages du terme ${ }^{6}$. Mis en circulation par Leibniz dès l'aube du siècle à propos des débats autour de Locke et sa philosophie de la connaissance, il devint rapidement une qualification infamante dans les mains des antiphilosophes. Entré en 1743 dans le Dictionnaire de Trévoux (d'obédience jésuite), il devint coextensif des notions d'athéisme et d'impiété, voire synonyme de complot contre le trône et l'autel; il pouvait embrasser indifféremment La Mettrie et Voltaire, Diderot et Rousseau, Quesnay et Montesquieu ; il ne s'embarrassait d'aucun distinguo entre matérialisme et déisme. Le matérialisme et le "spinozisme" apparaissent ainsi moins comme une revendication de philosophes réputés extrêmes (Diderot, d'Holbach, Helvétius) que comme une grave disqualification de l'ensemble du parti philosophique. Chaumeix résume bien la chose: «Je reproche aux encyclopédistes qu'ils sont matérialistes » (cité par Salaün 2010 : 37). Il s'agit d'un vocabulaire-clé pour la censure à la fois sociale et institutionnelle de l'Ancien Régime, qui pèse fortement sur la mise en forme des argumentations philosophiques. Franck Salaün n'en conclut pas que le matérialisme n'est qu'une invention des antiphilosophes, mais son analyse éclaire la dynamique des prises de position entre les camps opposés, 
les choix tactiques des uns et des autres pour répondre sur le fond comme sur la forme aux tentatives continuelles de disqualification : par le déni, l'usage des pseudonymes et le jeu sur l'ironie (Voltaire), l'affirmation d'un principe spirituel irréductible au corps (Rousseau), le déploiement alternatif d'un monisme vitaliste (Diderot), le retournement du stigmate (d'Holbach). Toutes ces subtilités infra-textuelles semblent échapper à la lecture de Jonathan Israël.

On se permet d'ajouter une remarque. Ce dernier a probablement raison quand il souligne l'intérêt teinté d'hostilité de Voltaire pour Spinoza dans la dernière phase de son existence, afin de combattre l'offensive matérialiste de Diderot, d'Holbach ou Helvétius. Il omet cependant de préciser que cet intérêt n'était pas entièrement neuf et que, après avoir longtemps dépeint Spinoza en athée vertueux et métaphysicien faux, mais conséquent, inoffensif au regard des ravages bien réels du fanatisme, Voltaire redoutait moins son héritage matérialiste en lui-même que sa réputation scandaleuse, propre à dissuader les soutiens du parti philosophique (Hazard 1941). La modération n'est autre, ici, qu'une mise en forme d'intérêts expressifs imposée par un système de censure bien réel. Comme le rappelait Turgot - autre cible d'Israël -, si la condamnation de l'Esprit (1758) par le Parlement de Paris était regrettable, c'est d'abord parce qu'elle faisait « tomber le poids réel sur beaucoup d'honnêtes gens de lettres qui reçoivent le fouet qu'Helvétius avait mérité » (Condorcet \& Turgot 1970 : 145). Protégé par son statut de fermier général, Helvétius restait à l'abri des risques que son audace fit encourir à d'autres.

14 Bertrand Binoche conclut son ouvrage sur cette jolie formule: «ce pluriel [des Lumières] est une merveille ». Ces trois ouvrages donnent aussi à voir une belle pluralité de pratiques en histoire de la philosophie. S'ils se focalisent sur les concepts, tous ne sauraient être assimilés à une exégèse idéaliste. Au contraire, ils sont susceptibles d'offrir une entrée précieuse pour saisir les enjeux sociopolitiques des Lumières et la structure de l'ordre symbolique, et pourraient être utilement confrontés à l'histoire sociale de la culture (Daniel Roche ou Robert Darnton) ou à la sociologie des idées proposée par Charles Camic (1983). Si la démarche de Jonathan Israël se place résolument en dehors de ce type d'approches, les travaux de Bertrand Binoche et de Franck Salaün entretiennent avec elles d'étonnantes correspondances. Sur la base d'une étude exclusivement centrée sur les argumentations philosophiques, la thèse centrale d'Écrasez l'infâme (les Lumières inaugurent un geste critique collectif à l'égard de la «superstition ») éclaire, en effet, l'homologie entre positions et prises de position, plus souvent postulée que démontrée, et en ce sens peut se lire comme une contribution à l'histoire sociale de la république des lettres. Celle-ci avait montré que le régime intellectuel des Lumières ${ }^{7}$ apparaît, grosso modo et au moins en France, comme le produit d'une alliance: celle entre une fraction des gens de lettres avec certaines franges de l'aristocratie contre un adversaire commun, l'Église et ses doubles (les parlementaires jansénistes, l'Université). Quant à L'Autorité du discours, son usage des sciences sociales demeure un peu impressionniste, mais il offre de nombreux indices sur l'encastrement des hommes des Lumières dans les réseaux de pouvoir, leurs formes de légitimation réciproques, ou sur la mobilité des frontières séparant les «philosophes » de leurs adversaires. Une fois écartées les facilités essentialistes - un « esprit » des Lumières ou un contenu doctrinal déterminé et universellement partagé -, la question tant débattue de la définition des Lumières reste ouverte. La perspective relationnelle de ces contributions permet néanmoins de tracer les contours d'un style de pensée, ce qu'on a appelé plus haut un agencement différentiel de prises de position: la 
pluralité des Lumières n'y dissout pas leur mouvement d'ensemble, celui d'un grand mouvement de dé-théologisation qui porta bien au-delà du cercle des philosophes parisiens ou de l'Encyclopédie. Les conséquences intellectuelles furent considérables. La philosophie des Lumières inventa une théorie sociale autonome qui ouvrit la voie à la sociologie et à l'ethnologie, réaffirma l'économie politique, inaugura une théorie et une pratique de l'histoire attentives au temps long de la démographie et de la production plutôt qu'aux intrigues de cours et aux récits de bataille. Et l'on ne mentionne ici que les sciences humaines et sociales.

CONDORCET Jean-Antoine-Nicolas de Caritat de \& TURGOT Anne-Robert-Jacques (1970).

Correspondance inédite de Condorcet et de Turgot : 1770-1779. Genève, Slatkine Reprints, éd. par

Charles Henry.

\section{BIBLIOGRAPHIE}

BELISSA Marc (2006). « Les Lumières radicales. La Philosophie, Spinoza et la naissance de la modernité (1650-1750) ». Annales historiques de la Révolution française, 345 : 204-208.

BINOCHE Bertrand (2007). La Raison sans l'histoire : échantillons pour une histoire comparée des philosophies de l'histoire. Paris, Presses universitaires de France.

BINOCHE Bertrand (2013). Les Trois sources des philosophies de l'histoire: 1764 - 1798. Paris, Hermann. BINOCHE Bertrand (2018). Nommer l'histoire : parcours philosophiques. Paris, Éditions EHESS.

CAMIC Charles (1983). Experience and Enlightenment : Socialization for Cultural Change in EighteenthCentury Scotland. Edinburgh, Edinburgh University Press.

CASSIRER Ernst (2006) [1932]. La Philosophie des Lumières [Die Philosophie der Aufklärung]. Traduit de l'allemand par Pierre Quillet. Paris, Fayard.

Foucault Michel (1984). « Qu'est-ce que les Lumières ? ». In Foucault Michel. Dits et écrits. Paris, Gallimard, 1994, t. IV, $339:$ 562-578.

GAY Peter (1977). The Enlightenment : an Interpretation. New York, Norton, 2 vol.

HAZARD Paul (1941). « Voltaire et Spinoza ». Modern Philology, 38(3) : 351-364.

HEILBRON Johan (2008) [1991]. Naissance de la sociologie [Het ontstaan van de sociologie]. Traduit du néérlandais par Paul Dirkx. Marseille, Agone.

ISRAËL Jonathan (2005) [2001]. Les Lumières radicales, la philosophie, Spinoza et la naissance de la modernité (1650-1750) [Radical Enlightenment : Philosophy and the Making of Modernity, 1650-1750]. Traduit de l'anglais par Pauline Hugues, Charlotte Nordmann et Jérôme Rosanvallon. Paris, Éditions Amsterdam.

ISRAËL Jonathan (2008). Enlightenment Contest : Philosophy, Modernity, and the Emancipation of Man 1670-1752. Oxford, Oxford University Press.

ISRAËL Jonathan (2011). Democratic Enlightenment : Philosophy, Revolution, and Human Rights, 1750-1790. Oxford, Oxford University Press. 
ISRAËL Jonathan (2019) [2014], Idées révolutionnaires : une histoire intellectuelle de la Révolution française [Revolutionary Ideas : an Intellectual History of the French Revolution from "The Rights of Man" to Robespierre]. Traduit de l'anglais par Marc-Olivier Bherer. Paris, Buchet-Chastel.

JAСоB Margaret C. (2006). The Radical Enlightenment : Pantheists, Freemasons, and Republicans.

Lafayette, Cornerstone Book.

LILTI Antoine (2009). « Comment écrit-on l'histoire intellectuelle des Lumières? ». Annales.

Histoire, Sciences Sociales, 64(1) : 171-206.

LILTI Antoine (2019). L'Héritage des Lumières : ambivalences de la modernité. Paris, Gallimard/Seuil, « Hautes études ».

REDIKER Marcus (2019). Un Activiste au temps des Lumières : le destin singulier de Benjamin Lay. Traduit de l'anglais par Aurélien Blanchard. Paris, Seuil.

ROZA Stéphanie (2015). Comment l'utopie est devenue un programme politique : du roman à la Révolution. Paris, Classiques Garnier.

SALAÜN Franck (1996). L'Ordre des mœurs : essai sur la place du matérialisme dans la société française du XVIII ${ }^{e}$ siècle, 1734-1784. Paris, Editions Kimé.

TACKETT Timothy (1997). Par la volonté du peuple : comment les députés de 1789 sont devenus révolutionnaires. Traduit de l'anglais par Alain Spiess. Paris, Albin Michel.

\section{NOTES}

1. Pour une recension plutôt favorable, voir Marc Belissa 2006.

2. On en trouvera un développement assez complet chez Antoine Lilti 2009. Ce dernier a aussi fait paraître une importante contribution à la question de la définition des Lumières : la place manque ici pour en rendre compte (Lilti : 2019).

3. L'expression est empruntée à l'historienne des sciences Margaret C. Jacob 2006.

4. Toutefois l'historien persiste et signe dans son ouvrage consacré aux idées des révolutionnaires, voir Jonathan Israël 2019.

5. On pense en particulier à l'abbé Morelly et l'abbé Mably, comme l'a montré Stéphanie Roza (2015).

6. L'auteur est un spécialiste du matérialisme des Lumières, voir en particulier Franck Salaün 1996.

7. Pour reprendre une expression de Johan Heilbron (2008), qui évite les apories de la transposition du concept de champ à l'Ancien Régime.

\section{AUTEURS}

\section{ARNAULT SKORNICKI}

Université Paris Nanterre/Institut des sciences sociales et du politique (ISP) 